INLO-PUB-17/96

\title{
$\underline{\text { Square Symanzik action to one-loop order }}$
}

\author{
Jeroen Snippe \\ Instituut-Lorentz for Theoretical Physics, \\ University of Leiden, PO Box 9506, \\ NL-2300 RA Leiden, The Netherlands.
}

Abstract: We present the one-loop coefficients for an alternative Symanzik improved lattice action with gauge groups $\mathrm{SU}(2)$ or $\mathrm{SU}(3)$.

Recently a new improved lattice action, called the square Symanzik action, was introduced by adding a $2 \times 2$ Wilson loop to the Lüscher-Weisz Symanzik action [1, 201:

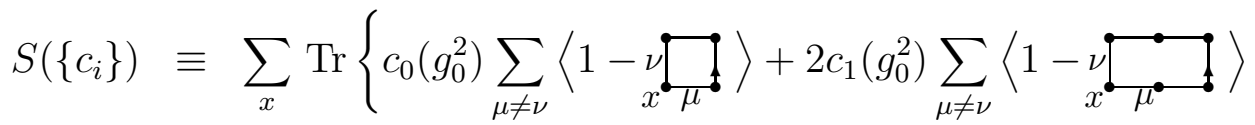

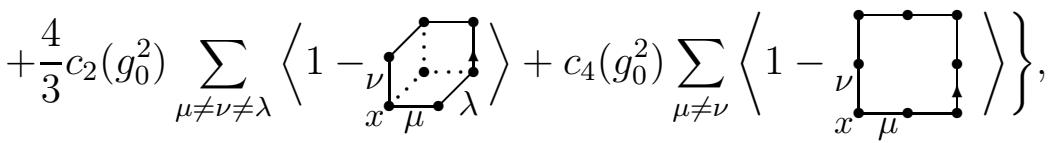

where the $<>$ imply averaging over the two opposite directions for each of the links. The inclusion of the $2 \times 2$ loop allows a simple diagonalization of the gauge field propagator, provided one takes $c_{0} c_{4}=c_{1}^{2}$ (where $c_{i} \equiv c_{i}\left(g_{0}^{2}=0\right)$ ). This simplifies certain analytic calculations, even if tadpole corrections [3] are incorporated. For details we refer to ref. [4].

The aim of Symanzik improvement is to cancel leading $\left(\mathcal{O}\left(a^{2}\right)\right)$ corrections in the lattice spacing $a$. The simplest choice for (on-shell) Symanzik improvement at tree-level amounts to [2]

$$
c_{0}=5 / 3, \quad c_{1}=-1 / 12, \quad c_{2}=0, \quad c_{4}=0 .
$$

For the square action one takes instead [4]

$$
c_{0}=16 / 9, \quad c_{1}=-1 / 9, \quad c_{2}=0, \quad c_{4}=1 / 144,
$$

which satisfies $c_{0} c_{4}=c_{1}^{2}$. At tree-level many other Symanzik improved actions can be easily constructed. This freedom has been used, e.g. in ref. [0], to study the universality of improvement by comparing the effectiveness of alternative actions.

Up to now there has been only one choice of the improvement coefficients, eq. (2), for which a one-loop calculation was completed [2]. Here we present our results of a one-loop calculation belonging to the square Symanzik action, eq. (35). Details of our calculation, that is based on the methods of Lüscher, Weisz and Wohlert [2, 6], will be presented 


\begin{tabular}{|c|cc||cc|}
\hline & \multicolumn{2}{|c||}{ Lüscher-Weisz } & \multicolumn{2}{c|}{ square } \\
\hline$\tilde{c}_{0}^{\prime}$ & $0.135160(13)$ & $(N=2)$ & $0.113417(11)$ & $(N=2)$ \\
& $0.23709(6)$ & $(N=3)$ & $0.19320(4)$ & $(N=3)$ \\
\hline$\tilde{c}_{1}^{\prime}$ & $-0.0139519(8)$ & $(N=2)$ & $-0.0112766(7)$ & $(N=2)$ \\
& $-0.025218(4)$ & $(N=3)$ & $-0.019799(2)$ & $(N=3)$ \\
\hline$c_{2}^{\prime}$ & $-0.0029431(8)$ & $(N=2)$ & $-0.0029005(7)$ & $(N=2)$ \\
& $-0.004418(4)$ & $(N=3)$ & $-0.004351(2)$ & $(N=3)$ \\
\hline$\Lambda / \Lambda_{\text {Wilson }}$ & $4.1308935(3)$ & $(N=2)$ & $4.0919901(2)$ & $(N=2)$ \\
& $5.2921038(3)$ & $(N=3)$ & $5.2089503(2)$ & $(N=3)$ \\
\hline$w(1,1)$ & $0.366262680(2)$ & $0.3587838551(1)$ \\
\hline$w(1,2)$ & $0.662626785(2)$ & $0.6542934512(1)$ \\
\hline$w(2,2)$ & \multicolumn{2}{|c||}{$1.098143594(2)$} & $1.0887235337(1)$ \\
\hline
\end{tabular}

Table 1: One-loop improvement coefficients $c_{i}^{\prime}$, defined by $c_{i}\left(g_{0}^{2}\right)=c_{i}+c_{i}^{\prime} g_{0}^{2}+\mathcal{O}\left(g_{0}^{4}\right)$, for the Lüscher-Weisz and square Symanzik actions. $N$ is the number of colors, while $\tilde{c}_{0}^{\prime}$ and $\tilde{c}_{1}^{\prime}$ stand for $c_{0}^{\prime}-16 c_{4}^{\prime}$ and $c_{1}^{\prime}+4 c_{4}^{\prime}$ respectively. For completeness we include the Lambda parameter ratios and the expectation values of a few $a \times b$ Wilson loops, $\left\langle N^{-1} \operatorname{Re} \operatorname{Tr} U(a \times b)\right\rangle \equiv 1-\frac{1}{4} g_{0}^{2}\left(N-N^{-1}\right) w(a, b)+\mathcal{O}\left(g_{0}^{4}\right)$. By convention the tadpole parameter $u_{0}$ equals $\left\langle N^{-1} \operatorname{Re} \operatorname{Tr} U(1 \times 1)\right\rangle^{1 / 4}$.

elsewhere. Introducing the notation $c_{i}\left(g_{0}^{2}\right)=c_{i}+c_{i}^{\prime} g_{0}^{2}+\mathcal{O}\left(g_{0}^{4}\right)$ we refer to table 1 for the coefficients $c_{i}^{\prime}$. At this point we stress that $c_{4}^{\prime}$ is a free parameter because in the expansion of the action to $\mathcal{O}\left(a^{2}\right)$ only the combinations $\tilde{c}_{0}\left(g_{0}^{2}\right) \equiv c_{0}\left(g_{0}^{2}\right)-16 c_{4}\left(g_{0}^{2}\right)$ and $\tilde{c}_{1}\left(g_{0}^{2}\right) \equiv c_{1}\left(g_{0}^{2}\right)+4 c_{4}\left(g_{0}^{2}\right)$ contain $c_{4}\left(g_{0}^{2}\right)$.

The following checks were performed to convince ourselves of the validity of the results in table 11 .

- For the Lüscher-Weisz action all results of the original calculation [2, 6] were reproduced, in most cases to a slightly higher accuracy. Especially the agreement with ref. [2] is non-trivial because we used covariant, instead of coulomb, gauge fixing.

- Coefficients are extracted from physical quantities computed as a function of the lattice spacing. We checked that divergences cancel, the one-loop beta function is reproduced, continuum limits are independent of the action chosen, and $a^{2} \ln (a)$ terms do not appear for the Lüscher-Weisz and square actions - as expected from Symanzik's analysis for $\varphi^{4}$ [1].

- The combination $\tilde{c}_{1}^{\prime}-c_{2}^{\prime}$ was computed both using the static quark potential method of ref. [6] and the twisted finite volume method of ref. [2]. The agreement is better than $0.003 \%$.

- Using three completely different methods: (a) static quark potential; (b) three point vertex in a twisted finite volume; (c) (for $\mathrm{SU}(2)$ ) a background field calculation in a periodic finite volume [4], the Lambda parameters extracted agree to at least six digits. 
We conclude with testing how well the tadpole correction [3] to the $\mathrm{SU}(3)$ tree-level square Symanzik action predicts the one-loop correction. Since, to $\mathcal{O}\left(a^{2}\right), c_{4}\left(g_{0}^{2}\right)$ can be freely chosen, the relevant test is comparing

$$
\frac{\tilde{c}_{1}\left(g_{0}^{2}\right)}{\tilde{c}_{0}\left(g_{0}^{2}\right)}=-\frac{1}{20}\left(1+0.1217 g_{0}^{2}+\mathcal{O}\left(g_{0}^{4}\right)\right)
$$

to

$$
\frac{c_{1} u_{0}^{-2}+4 c_{4} u_{0}^{-4}}{c_{0}-16 c_{4} u_{0}^{-4}}=-\frac{1}{20}\left(1+0.0957 g_{0}^{2}+\mathcal{O}\left(g_{0}^{4}\right)\right) .
$$

Here $u_{0}=1-0.3588 g_{0}^{2} / 6$ was taken from table 11. It follows that the tadpole prediction captures $79 \%$ of the one-loop correction, a result similar to the $76 \%$ found for the Lüscher-Weisz Symanzik action. (For SU(2) one finds $80 \%$ for both actions).

Of course one may consider the ratios $c_{1}\left(g_{0}^{2}\right) / c_{0}\left(g_{0}^{2}\right)$ and $c_{4}\left(g_{0}^{2}\right) / c_{0}\left(g_{0}^{2}\right)$ separately. While for $c_{4}^{\prime}=0.003058$, satisfying $c_{4}\left(g_{0}^{2}\right) c_{0}\left(g_{0}^{2}\right)=\left(c_{1}\left(g_{0}^{2}\right)\right)^{2}$ to one-loop order, the tadpole prediction is off by $21 \%$ in both ratios, for $c_{4}^{\prime}=0.002401$ the deviations are only $11 \%$.

\section{Acknowledgments}

The author is grateful to Pierre van Baal and Margarita García Pérez for encouraging discussions.

\section{References}

[1] K. Symanzik, Nucl. Phys. B 226 (1983) 187, 205.

[2] M. Lüscher and P. Weisz, Phys. Lett. B 158 (1985) 250; Nucl. Phys. B 266 (1986) 309; Comm. Math. Phys. 97 (1985) 59; 98 (1985) 433 (E).

[3] G.P. Lepage and P.B. Mackenzie, Phys. Rev. D 48 (1993) 2250.

[4] M. García Pérez, J. Snippe and P. van Baal, hep-lat/9608036, submitted to Phys. Lett. B, and references therein.

[5] M. Alford, e.a., Phys. Lett. B 361 (1995) 87.

[6] P. Weisz and R. Wohlert, Nucl. Phys. B 236 (1984) 397; B 247 (1984) 544 (E). 